\title{
Row erupts over child aggression study
}

[WASHINGTON] Psychiatrists under attack for using the drug fenfluramine in a trial examining aggression in poor, non-white, innercity boys last week defended themselves, along with those who gave ethical approval to the experiments, against criticism appearing in the press.

Critics of researchers at the New York State Psychiatric Institute (NYSPI) in Manhattan claim that a paper published last year in the Archives of General Psychiatry shows the research was in flagrant breach of federal ethics rules governing research on children.

But the researchers say they obeyed those rules, and that the critics are using specious objections to cast doubt on the proper scientific investigation of aggressive behaviour. "The implication is that you should not study the relationship between aggression and biology," says Daniel Pine, a child psychiatrist and lead author of the paper, who describes the research as critical for helping to solve "a major public health problem".

B. Timothy Walsh, chair of the institute's Institutional Review Board (IRB), which approved the study - and is now under fire for having done so - says the panel correctly sought to interpret government ethics rules in the light of the facts of the protocol, not how it might be seen by critics.

"We feel we're being singled out somewhat unfairly," says Walsh, pointing out that other IRBs have approved studies using fenfluramine in children, and that the federal government regularly funds such research.

Pine and his co-authors reported a positive correlation between an indicator of fenfluramine-induced activity of serotonin in the central nervous system and both aggressive behaviour and social circumstances conducive to the development of such behaviour. The 34 boys studied, aged six to ten and either black or Latino, were the younger brothers of juvenile delinquents traced by investigators through court records.

Fenfluramine - part of the controversial diet drug combination 'Fen-Phen', which was withdrawn last year after it was discovered to cause heart valve damage — increases serotonin levels in the central nervous system. The New York trial, funded largely by the Lowenstein Foundation, with some support from the National Institute of Mental Health, was halted in 1995, two years before the drug was withdrawn.

Last week, the Long Island newspaper Newsday, followed by The New York Times and the New York Post, ran articles on the study quoting criticism from ethicists and others. The Office for Protection from Research Risks at the National Institutes of Health (NIH) has since confirmed that it is investigating protection of human subjects at the psychiatric institute.
For non-therapeutic research involving a "minor increase over minimal risk" — the category in which the IRB at the psychiatric institute considered the trial — federal rules say that children may be subjected only to experiences that are "reasonably commensurate" with their actual or expected medical situations. Also, the study must be likely to produce 'generalizable' knowledge about the subject's "disorder or condition" which is essential for understanding or improving it.

Disability Advocates, a public-interest law office in Albany, New York, which complained about the study to the NIH, contends that the children underwent experiences including overnight fasting, intravenous catheterization and oral administration of fenfluramine - beyond those that medically healthy children might reasonably expect.

It adds that because the children were healthy - a prerequisite for participation the study could not have yielded vital, generalizable knowledge on their "disorder or condition". CliffZucker, executive director of Disability Advocates, argues that researchers, the federal government and IRBs have all ignored "the clear rules about what's permissible and what's not".

But both Pine and Walsh say that many of the children were psychiatrically disturbed, and that all were at "high risk" of developing antisocial behaviour or aggression. This, they say, constitutes a "condition" under the federal rules.

Walsh argues that, in the IRB's view, "going to a medical setting, getting stuck with a needle and taking a pill that may make you feel a little funny" are "commensurate" with the experience of children without serious medical illnesses. Pine and Walsh say the IRB took care in approving the trial, referring it to a subcommittee with expertise on children and requiring extra documentation about fenfluramine's risks.

But others say the study had elements that should have worried the IRB. One was the racial composition of the group, which was 44 per cent black and 56 per cent Hispanic, a "highly disturbing" skew, according to Arthur Caplan, director of the Centre for Bioethics at the University of Pennsylvania.

Racial balance is particularly important in research with no direct benefit to subjects and that is of more than minimal risk, says Robert Levine, a professor of medicine who chairs Yale University's IRB.

The investigators have argued that their neighbourhood of New York City is largely Hispanic and black, and that they were seeking funding to expand recruitment to include whites when the trial was stopped for unrelated reasons.

Pine admits that the lack of white participants "was a flaw in our study", but only because it limited the generalizability of the findings. Caplan argues that potentially contentious work of this nature "will need to be taken to a different review level than the local institution". Such trials, he says, should be subject to a review body that "has not got the same pressures on it as the local IRB to get it approved".

MeredithWadman

\section{Rockets launch Spain's eye on the sky}

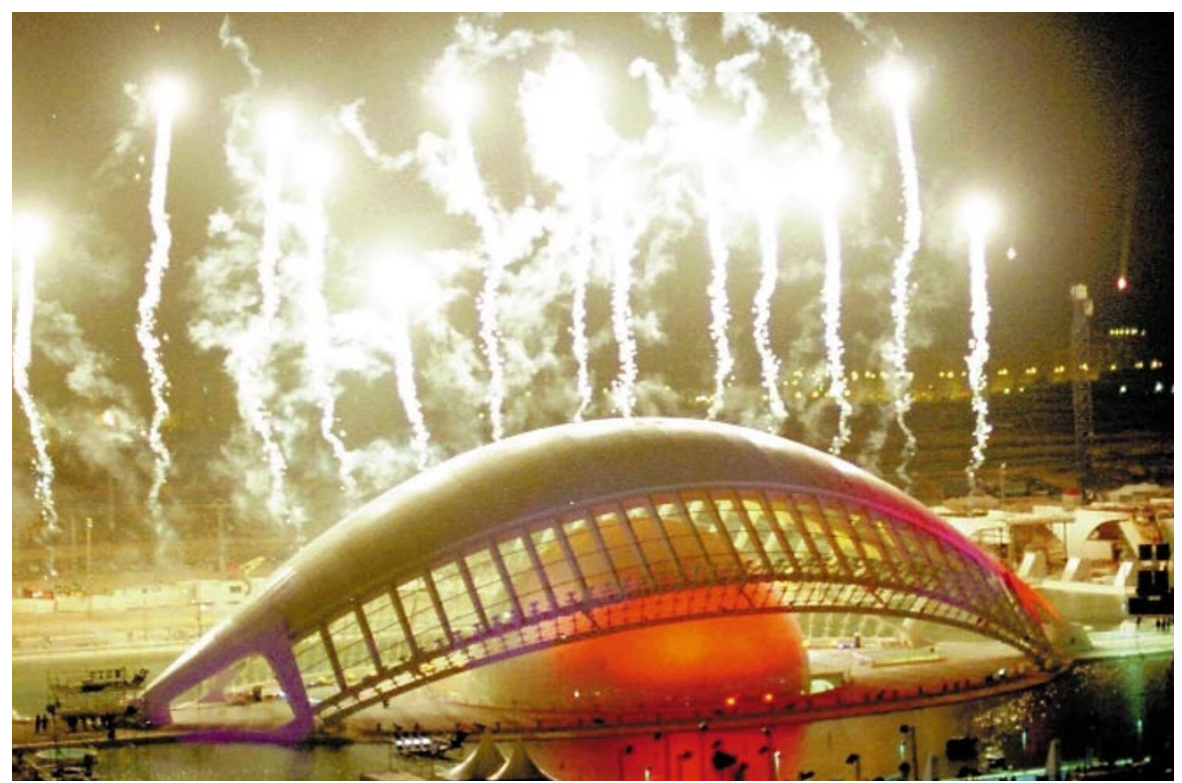

Fireworks lit up the sky last week at a ceremony to inaugurate a futuristic-looking planetarium in Valencia, eastern Spain. The planetarium, in the form of a human eye, was designed by Valencian architect Santiago Calatrava and forms part of a new art and science complex. 